\title{
Las mujeres, sus bienes y estado civil, entre costumbres y legislación. Las jefas de familia de la campaña de Buenos Aires de los siglos XVIII y XIX
}

\section{Claudia Contente*}

\section{Universitat Pompeu Fabra (UPF-GRIMSE)}

claudia.contente@upf.edu

Fecha recepción 28.11.2015 / Fecha aceptación 06.04.2016

\section{Resumen}

En el mundo hispanoamericano de los siglos XVIII y XIX el marco jurídico y el consenso social implicaban que mientras los hombres acaparaban el poder de actuar y decidir por la familia y sus bienes, las mujeres ocupaban -al menos idealmente- un lugar discreto, subordinado y sumiso pasando de la tutela del padre a la del marido, adquiriendo una total capacidad de acción en el caso de ser viudas.

Es bien sabido que ese ideal estaba lejos de reflejar la realidad, que las mujeres solían trabajar a la par de los hombres o por su cuenta

\begin{abstract}
In the Hispanic American world of the 18th and 19th centuries, the legal framework and social consensus meant that, while men monopolized the power to act and to decide on behalf of the family and their property, the role of women was, at least ideally, to be discreet, subordinate and submissive, moving only from the aegis of the father to that of the husband. They would only acquire full capacity to act were they to be widowed.

It is well known that this ideal did little to reflect reality. Women often worked alongside
\end{abstract}

\footnotetext{
* Trabajo realizado con el apoyo de la Agència de Gestió d’Ajusts Universitaris i de Recerca, SGR 1193 y del proyecto «Dentro y fuera: Cambio institucional e integración social y cultural en el Imperio Español contemporáneo, 15501950», Feder-Mineco HAR2015-68183-P.
} 
Monográfico | Las mujeres, sus bienes y estado civil, entre costumbres y legislación

y que sus respectivas vidas y situaciones conocían fuertes contrastes según los diversos sectores sociales.

Basándonos en la información que se desprende de los censos y repartos sucesorios en dos zonas de la campaña de Buenos Aires durante ese periodo, abordaremos la situación de las mujeres en general y en particular de las viudas que se encontraban al frente de su grupo doméstico: analizaremos un aspecto acotado, el de la relación con sus bienes y la capacidad concreta de que disponían según su estado civil para emprender acciones relacionadas con dichos bienes o decidir sobre su transmisión.

\section{Palabras clave}

estado civil, mujeres, bienes, transmisión, jefas de familia, campaña de Buenos Aires, siglo XIX. men or on their own, and sharp contrasts existed between the lives and situations of individuals according to their social background. Based on the information gleaned from censuses and succession arrangements in two areas of rural Buenos Aires during this period, we will address the situations faced by women - and particularly by widows who were heads of household. The analysis is limited to their relationships with their assets and their concrete capacity to undertake action related to the assets or to decide about their transfer, according to their marital status.

\section{Key words}

Keywords: marital status, women, assets, transmission, heads of household, countryside of Buenos Aires, 19th century. 
Se da por sentado que en el mundo Hispanoamericano de los siglos XVIII y XIX las mujeres solteras estaban sometidas a la autoridad y protección del padre o hermanos y una vez casadas pasaban a estarlo bajo la del marido. Los hombres eran quienes protegían, proveían los bienes y monopolizaban el criterio para decidir lo mejor para la familia, mientras que las mujeres, en un papel complementario y en buena medida subalterno del masculino, asumían la gestión del hogar. Era solamente en el eventual caso de encontrarse viudas que las mujeres tenían la capacidad efectiva para asumir decisiones y disponer de su vida y de sus bienes según su parecer. Si bien esto es incuestionable si se lo contempla a través de la ley escrita, tradiciones y costumbres, en los últimos años se ha puesto en evidencia que ese paradigma estaba lejos de reflejar el conjunto de la población, que muy probablemente se acerca a la realidad de las clases más favorecidas, especialmente en zonas urbanas, pero no habría sido tan estricto entre los sectores medios y bajos y menos aún en el mundo rural, donde las mujeres solían trabajar a la par de los hombres o por su propia cuenta ${ }^{1}$.

Para el caso concreto del Río de la Plata, desde hace unos años conocemos mejor cómo se estructuraban las familias y se organizaban sus actividades, cómo evolucionaban a lo largo de su ciclo de vital y cómo transcurría la vida de las mujeres, tanto entre las clases altas, -para las cuales abundan los testimonios sobre su papel en el hogar y la paulatina y progresiva participación en la esfera pública-, como en el mundo rural'2.

1. S. Mallo, "La mujer rioplatense a fines del siglo XVIII. Ideales y realidad", Anuario del IEHS V, Tandil, 1990, 117-132; C. Mayo, Estancia y Sociedad en La Pampa (1740-1820), Buenos Aires, 1995, 165-190.

2. Es muy amplia la bibliografía, a título indicativo. A propósito de la condición jurídica de la mujer, ver J. M. Ots Capdequí, "El sexo como circunstancia modificativa de la capacidad jurídica en nuestra legislación de Indias", en Anuario de Historia del Derecho Español, VII, 1930, 311-380; V. Kluger, "El rol femenino en el litigio familiar. Ajustes y desajustes, conformismo y contradicción en los pleitos familiares en el Río de la Plata”. Iberoamericana. América Latina, España, Portugal: Ensayos sobre letras, historia y sociedad, 
El trabajo pionero de Silvia Mallo a propósito de las mujeres porteñas a fines del siglo XVIII marcó un hito al mostrar, basándose en periódicos y fuentes judiciales, como las mujeres escapaban a menudo a ese papel que la sociedad patriarcal les atribuía. Las investigaciones de Carlos Mayo nos proporcionan algunas pautas a propósito de las actividades, en particular de las estancieras del mundo rural rioplatense, a partir de las cifras reflejadas por los censos del siglo XVIII y testimonios recogidos en fuentes judiciales. Nos proponemos aquí completar este panorama abordando otras perspectivas relacionadas con la familia y el lugar de las mujeres en aquel mundo campesino, en un ámbito muy acotado, el derivado de su estado civil, sus eventuales recursos cuando se encontraban al frente de la unidad familiar y más particularmente, su relación con sus bienes y consecuente capacidad de transmisión. Para este análisis nos basaremos fundamentalmente en la información contenida en los censos realizados en 1813 y 1815 para los cuales hemos procesado y analizado las cédulas censales y diversos testimonios que se deprenden de los repartos sucesorios.

La primera mitad del siglo XIX fue un periodo particularmente agitado en la campaña bonaerense, tras los sucesos que marcarían en 1810 el comienzo de la ruptura del vínculo colonial con España, empezaría un largo periodo de tensiones y guerras civiles que tendrían un alto costo en vidas y recursos materiales. Sin embargo, desde el punto de vista de los indicadores económicos, los eventos de orden político no tuvieron para las familias consecuencias catastróficas, al menos no tanto como lógicamente se podría presumir. Gracias a la creciente demanda internacional de productos agrícolas para cuya producción la región pampeana brindaba condiciones óptimas (especialmente de vacunos y luego hacia mediados de siglo de lana y ovinos), la zona siguió atrayendo migrantes, las familias fueron prosperando mientras se iban incorporando nuevos territorios al naciente estado a fuerza de hacer retroceder la frontera con los indios. En otros términos, pese al contexto local desfavorable, la economía siguió creciendo en parte gracias a la capacidad de adaptación de las familias, sobre las que reposaba esencialmente la producción agrícola.

La burocracia colonial, presente hasta 1810 , era prácticamente inexistente en amplias zonas de la campaña bonaerense. Durante el periodo colonial estaba representada por alcaldes de la hermandad, -vecinos elegidos para ejercer ese cargo por los propios habitantes del pueblo- ${ }^{3}$, que seguirían siendo los representantes de la justicia local durante las primeras

No 14, 2004, 7-28; sobre la participación en la esfera pública, que en las clases altas urbanas se habría producido principalmente desde su condición de esposas y madres organizando asociaciones caritativas y de beneficencia, ver por ejemplo, P. García Jordán y G. Dalla-Corte Caballero, "Mujeres y sociabilidad política en la construcción de los Estados Nacionales", en I. Morant (Dir.), Historia de las Mujeres en España y Latinoamérica, Vol. III, Del siglo XIX a los umbrales del XX, Madrid, 2006, 559-607; M. Bonaudo, "Cuando las tuteladas tutelan y participan. La Sociedad Damas de Caridad (1869-1894)”, Signos Históricos, núm. 15, enero-junio, 2006, 70-97; En cuanto al ámbito rural rioplatense más precisamente ver ante todo el trabajo pionero de A propósito de las mujeres de las clases altas rurales, consultar Y. de Paz Trueba, Mujeres y esfera pública. La campaña bonaerense entre 1880 y 1910, Rosario, 2010.

3. J. C. Garavaglia "La Justicia rural en Buenos Aires durante la primera mitad del siglo XIX (Estructuras, funciones y poderes locales)", en J. C. Garavaglia, Poder, conflicto y relaciones sociales. El Río de la Plata, XVIII-XIX, Buenos Aires, 1999, 89-121. 
décadas del periodo independiente. La justicia era primordialmente oral e incluso en algunos casos los propios alcaldes de la hermandad no sabían leer y escribir: los documentos a nuestra disposición referentes a los pobladores de este universo de los tiempos de la colonia y primeras décadas independientes son en consecuencia poco abundantes y más bien avaros en información en términos generales y, tal como es previsible, aún más en lo que se refiere a las mujeres. No hay que perder de vista que durante esos primeros tiempos de la gestación del estado, enfrentando guerras en diversos frentes y otros tipos de amenazas, las autoridades tendrían ciertamente otras prioridades más urgentes que llevar cuenta de los habitantes y sus actividades. La organización del estado y sus instituciones iría evolucionando paulatinamente y con ese proceso, aumentarían igualmente los documentos a nuestra disposición para ayudarnos a comprender la sociedad del periodo.

Referirnos a las mujeres campesinas, a su condición al frente de un grupo doméstico y a su patrimonio en estas alejadas regiones del antiguo Imperio español que era la campaña bonaerense conlleva inevitablemente referirnos al trabajo, a los medios con que podían contar para subsistir, evitando así de paso la noción a menudo implícita de que aquellas que tenían bienes, los habían recibido en herencia. ${ }^{4}$

Si a través de fuentes tributarias, censos o conflictos podemos reconstruir más o menos afortunadamente las actividades productivas de la zona, las mujeres quedaban casi sistemáticamente difuminadas en el conjunto de las actividades domésticas que, en el mejor de los casos, se atribuían al jefe de familia y a través de él, implícitamente al grupo doméstico. En los registros parroquiales no se solían inscribir las eventuales actividades que pudieran desarrollar ellas y los censos por regla general omitían también este aspecto englobando las actividades de la unidad e inscribiendo de vez en cuando una ocupación para aquellas que se encontraban al frente del grupo doméstico que, según las fuentes nominativas, eran casi indefectiblemente, viudas.

En ese sentido el trabajo femenino era parte de lo habitual y cotidiano, sería obvio y probablemente irrelevante para quienes vivían en aquella sociedad: no habría por qué consignar por escrito y menos aún conservar lo que quizás se escribiera sobre algo que resultaba evidente. Sin embargo algunas pistas nos permiten reconstruir estos aspectos.

\section{Las mujeres y sus actividades}

En los años 1813-1815 la nueva administración organizó un relevamiento de población para el conjunto de la campaña de Buenos Aires a fin de conocer la capacidad de la región para proveer recursos para las guerras de independencia que se estaban librando en esos momentos ${ }^{5}$.

4. O. Rey Castelao, "Mujeres en la economía campesina”; en I. Morant, (dir.), Historia de las mujeres en España y América Latina, vol II, Madrid, 2005, 263-286.

5. A propósito de estos censos en su conjunto ver GIHRR, "La sociedad rural bonaerense a principios del siglo XIX. Un análisis a partir de las categorías ocupacionales”, en R. Fradkin y J. C. Garavaglia, (editores); En busca de un tiempo perdido. La economía de Buenos Aires en el país de la abundancia 1750-1865, Buenos Aires, 2004, 21-63. 
Estos censos de principios del siglo XIX no mencionan prácticamente actividades para ellas. Si bien las mujeres sin duda trabajaban tanto en sus hogares como fuera de ellos, tenemos pocos datos concretos al respecto, se les atribuye una actividad en el caso que eventualmente se encontraran como jefas de familia y, aun así, algunos censistas podían tener más tendencia a obviar sus actividades que en el caso de los hombres ${ }^{6}$.

Es solo a partir de 1869, cuando se realiza el Primer Censo Nacional, que comenzarán a registrarse de manera casi sistemática las actividades con que las mujeres se ganaban la vida: al confeccionar el primer censo nacional, se pedirá a los censistas que completen la ocupación de todas y cada una de las personas mayores de 14 años que inscribían ${ }^{7}$, esos son los primeros datos medianamente fiables con que contamos sobre el trabajo femenino y que abarcan al conjunto de la población ${ }^{8}$.

Sin sorpresas, constatamos que tanto en los pueblos rurales como en las zonas puramente agrícolas, la gran mayoría de las mujeres encontraba una ocupación en las tareas domésticas como las de lavandera, costurera, etc., actividades que podían realizarse en la casa y permitían conciliar el cuidado de la familia con una actividad remunerada9 ${ }^{9}$

Era en los pueblos donde se instalaban preferentemente las solteras y viudas. Allí la mayor concentración de habitantes ofrecía mayores posibilidades, tanto para ganarse el sustento como de integrarse en una red de solidaridades más sólida y nutrida ${ }^{10}$. Y si bien las tareas domésticas predominaban ampliamente, existía asimismo la posibilidad de trabajar en los comercios al menudeo o en toda una gama de tareas artesanales como tejedoras, bordadoras

6. En la zona de La Matanza todas las viudas al frente de una explotación constan como criadoras de ganado o labradoras. En San Vicente el 52\% de las mujeres que son jefas de familia no tienen una ocupación atribuida, mientras que en el caso de los hombres no se declara la actividad de un $9 \%$ de los jefes de familia. Dada la calidad de los datos, hemos optado por presentar los correspondientes a las jefas de familia viudas de La Matanza.

7. Se intentaba conocer mejor los habitantes y comprender lo que cada uno de ellos podía aportar a ese estado que se estaba construyendo, H. Otero, Estadística y Nación. Una historia conceptual del pensamiento censal de la Argentina Moderna. 1869-1914, Buenos Aires, 2006.

8. Se podrían mencionar igualmente los relatos de viajeros, sin embargo los testimonios de este tipo son particularmente parcos. Estos autores solían escribir teniendo en mente su patria de origen y comparando sistemáticamente con lo que veían, con lo cual suelen resultar más elocuentes a propósito de sus orígenes y de lo que hoy veríamos como sus propios prejuicios que sobre el mundo que estaban describiendo. Cf. C. Mayo, Estancia y Sociedad... op.cit. 165-166; H. Jochims Reichel, "La mujer rioplatense en la visión de los viajeros: un sujeto de historia”, J. Andreo y S. B. Guardia, Historia de las mujeres en América Latina, Murcia, 2002, 181-193.

9. C. Contente; M. F. Barcos, "La parte sumergida del iceberg. Mujeres trabajadoras en la campaña de Buenos Aires (Argentina) según el Primer Censo Nacional de Población de 1869”, T. M. Ortega López (ed), Jornaleras, Campesinas y Agricultoras. La Historia Agraria desde una perspectiva de género, Zaragoza, 81109.

10. Se trata de un fenómeno bien conocido, ver por ejemplo: A. Fauve-Chamoux, "Le surplus urbain des femmes en France préindustrielle et le rôle de la domesticité", Population, 53e année, no 1-2, 1998, 359-377 o O. Hufton, "Women Without Men: Widows and Spinsters in Britain and France in the Eighteenth Century", en Journal of Family History, 1984, vol. 9, 4, 355-376. 
o cigarreras que también se concentraban de preferencia en estos pueblos rurales o en ejidos. Las tareas agrícolas, en la mayoría de los casos en relación de dependencia, constituían otra de las posibilidades laborales en el medio rural, predominando en este caso las mujeres casadas, las «amancebadas»-conviviendo en unión ilegítima- o, eventualmente, acompañadas por alguna otra mujer. Muchas de ellas aparecen al frente de explotaciones agrícolas, ya sean orientadas al cultivo de cereales o a la cría de ganado, -esencialmente en el ámbito rural, aunque también había algunas mujeres en estas circunstancias en los pueblos al frente de algún comercio- En 1869 detectamos esta situación exclusivamente en el caso de estar viuda o el marido ausente: ya en ese entonces, sería prácticamente imposible para una mujer hacerse un lugar al frente de una explotación o comercio sola y por sus propios medios.

Otro aspecto cuya evolución muestran los censos entre principios del siglo XIX y el censo de 1869 es el notable aumento de la densidad de población, debida principalmente a un fuerte flujo migratorio ${ }^{11}$. Este incremento del número de habitantes, acompañado por la llegada de inversores de otros horizontes, implicó la proletarización de muchos campesinos y una situación más difícil para las familias: si a principios de siglo el aporte que ellas ofrecían a la economía doméstica podía representar un complemento para los ingresos hogareños, en la segunda mitad del siglo, ese ingreso se habría vuelto indispensable para el sostén del grupo ${ }^{12}$.

Podemos constatar así en la zona de San Vicente en 1869, que mientras la mitad de las viudas y un tercio de las solteras declaraban una actividad remunerada, esta proporción se reducía en el caso de las casadas, entre las cuales solo una de cada siete consignaba una ocupación.

\section{Mujeres jefas de familia}

Los censos de 1813 y $1815^{13}$, contrariamente a los que se registrarían posteriormente, tienen la ventaja de incluir a todos los miembros de la unidad y marcar divisiones entre las familias, designando quiénes son las personas que están compartiendo la residencia «bajo el mismo

11. La absoluta falta de precisión en la definición de la superficie cubierta por los censos de principios del siglo XIX, sumado a los repetidos cambios en las jurisdicciones administrativas, hacen que sea imposible estimar ni siquiera de forma aproximada el aumento para cada zona. A título indicativo la superficie de San Vicente habría tenido según R. Levene unos $8.000 \mathrm{~km}^{2}$ hasta 1822 en que tras la creación de varios partidos sucesivamente (a su costa y de la de otros partidos que lo rodeaban) se habrían convertido en unos 1.200 km2 para 1869. Cf. R. Levene, Historia de la Provincia de Buenos Aires y de la formación de sus pueblos, La Plata, 1941, 641-646 y Registro Estadístico de la Provincia de Buenos Aires, Publicación Oficial, Buenos Aires, 1869.

12. C. Contente, "Percevoir l'invisible : le travail des femmes à la campagne de Buenos Aires du XIXème siècle à partir du recensement de 1869", en M.-P. Arrizabalaga; I. Bolovan, M. Eppel; J. Kok; M. L. Nagata (Coords.) Many Paths to Happiness? Studies in Population and Family History. A Festschrift for Antoinette Fauve-Chamoux, Amsterdam, 2010, 81-95.

13. Archivo General de la Nación, Buenos Aires (en adelante, AGN) X-8-10-4. 
techo y al mismo fuego», lo que nos permitirá detenernos a observar rápidamente la composición de los grupos encabezados por mujeres en dos zonas rurales cercanas a la ciudad de Buenos Aires, La Matanza y San Vicente.

La Matanza era ya a principios del siglo XIX una zona donde, gracias a la corta distancia a Buenos Aires, la producción de cereales y ganado era altamente rentable y se podían obtener buenos salarios, mientras San Vicente, a $50 \mathrm{~km}$ al sur de la ciudad de Buenos Aires, era un área de frontera abierta con los indígenas, donde eran particularmente los pequeños campesinos quienes protagonizaban el avance de la frontera, instalándose y colonizando de a poco el territorio indígena. Con el paso del tiempo el control de la zona se fue consolidando y San Vicente también se convirtió en una zona segura para instalarse.

El censo de La Matanza en 1813 designa, entre sus 211 agregados domésticos, nueve unidades encabezadas por mujeres. El de San Vicente en 1815, con sus 766 unidades familiares, cuenta con una proporción más importante: 78 explotaciones constan bajo la responsabilidad de una mujer.

Podemos presumir la ausencia temporaria del marido de Bernarda Chávez, la única casada al frente de una unidad en La Matanza, las otras mujeres presentes, todas ellas viudas, reúnen varios puntos en común: tienen más de 40 años, hijos ya mayores (de más de 20 o 25 años) y eventualmente también esclavos y/o peones. Hay solo un caso de una viuda más joven, Paula Guisande, de 35 años, con niños pequeños bajo su responsabilidad (de entre 4 y 13 años), en cuya unidad conviven igualmente dos esclavos y tres peones. Cerca de la unidad de Paula residen otros miembros de su familia y de la de su difunto esposo.

Resumiendo, en estas unidades encabezadas por viudas en La Matanza residen entre 10 y 16 miembros -el promedio general de habitantes por explotación familiar se situaba, según ese censo, entre 7 y 7,5 miembros-. Salvo en este último caso, los hijos están en edad de participar activamente en las tareas de la explotación e incluyen peones y esclavos. En otras palabras, ellas siguen al frente de la unidad que genera suficientes recursos para procurarse la mano de obra exterior al grupo que fuera necesaria: poseían un patrimonio consolidado y la presencia de hijos que participaban en las actividades, representaban la perspectiva de continuidad a mediano o largo plazo de la explotación y su trasmisión a la siguiente generación ${ }^{14}$.

El censo declara seis viudas con hijos pequeños que conviven con sus propios padres o hermanos -en algunos casos con la mención «agregadas», mientras que en otras seis explotaciones la unidad acoge a la madre o la suegra (viudas) del jefe de familia o de su esposa.

Evidentemente, si los niños eran pequeños y el patrimonio demasiado exiguo como para poder compensar la falta del padre con esclavos o jornaleros, la solución era «agregarse» en la casa que estuviera dispuesta a acogerlos, quizás hasta que la viuda volviera a casarse, o que los hijos crecieran y se independizaran.

14. A propósito de las posibles estrategias que se ofrecían a una viuda consultar M. Oris y E. Ochiai "Family Crisis in the Context of Different Family Systems: Frameworks and Evidence on When Dad Died" en R. Derosas y M. Oris (ed.) When Dad Died: Individuals and Families Coping with Distress in Past Societies, Bern, 2002, 17-79. 
El censo de San Vicente, pese a los escasos $60 \mathrm{~km}$ que separan una zona de la otra, muestra una realidad bien diferente. El espacio allí aún no está saturado, hay más posibilidades de instalarse por su cuenta, y hay mayor proporción de mujeres al frente de la unidad. Las situaciones familiares son igualmente mucho más diversas que las registradas en La Matanza. Entre las 78 jefas de familia mujeres, se cuentan cinco casadas y, un hecho notable, dos solteras "criadoras», una de ellas es María Quiroga, madre soltera con sus dos hijos de $10 \mathrm{y}$ 8 años y dos peones o Mercedes Ávila, que llevaba adelante sola su explotación conviviendo con una esclava y dos peones en tierras arrendadas y sin ningún otro pariente bajo su techo ni instalado a proximidad.

Contrariamente a La Matanza, en San Vicente encontramos unidades encabezadas por viudas con niños a veces pequeños, recogiendo en algunos casos a otras personas, a veces a otras viudas, a «peones agregados» ${ }^{15} \mathrm{o}$ a familias completas «agregadas» en la unidad, de modo de poder reunir la mano de obra indispensable para poder si no mejorar, al menos sobrevivir «arañando la tierra».

Muy probablemente el alto rendimiento agrícola en La Matanza hacía que la explotación fuera más intensiva, la competencia también y que para las mujeres hubieran menos oportunidades de encontrarse solas al frente de una explotación. La falta de registros parroquiales para La Matanza, impide verificar la eventual incidencia de las segundas nupcias, un factor que probablemente explique esta situación.

Los censos posteriores, realizados en 1836 y $1838^{16}$, comportan en cambio solo los nombres de los jefes de familia acompañados por el número y la etnia de las personas que comparten la unidad sin mencionar el estado civil. La comparación de los datos censales de dos zonas de la campaña de Buenos Aires, Matanza y San Vicente ${ }^{17}$, delatan claramente que la proporción de unidades encabezadas por mujeres aumentan de manera sensible entre 1813 y $1836 / 1838$, lo que parece ser una tendencia general en la provincia de Buenos Aires ${ }^{18}$. Las razones de este aumento proporcional de las mujeres como jefas de familia son diversas y están ligadas principalmente con la guerra y con procesos migratorios en búsqueda de nuevas y mejores oportunidades.

15. Se entiende que los «peones agregados» a diferencia de los «peones» a secas no recibían un salario si no que participaban en un porcentaje de los beneficios y pérdidas, recibiendo eventualmente casa y comida.

16. AGN X-25-2-4 y AGN X-25-6-2

17. Para más detalles sobre estas regiones, cf. C. Contente, Familias en la Tormenta. Tierra, familia y transmisión de patrimonio en el Río de la Plata, siglos XVIII y XIX, Buenos Aires, 2015, 1ra parte.

18. Mientras que en La Matanza las mujeres jefes de familia pasan de 4,2\% en 1813 a 11,3\% en 1838 . En San Vicente y Ranchos se verifican tendencias similares, ellas son 10,7\% de los que encabezan una familia en 1815 , en San Vicente son $15,4 \%$ en 1836 y 13,8\% en 1838. En otras zonas como Azul los porcentajes son del mismo orden: las mujeres al frente de una unidad son $14,5 \%$. cf. S. Lanteri Un vecindario federal. La construcción del orden rosista en la frontera sur de Buenos Aires. Un estudio de caso (Azul y Tapalqué), Tesis Universidad del Centro de la Provincia de Buenos Aires, 2008, p. 225; G. Banzato, La expansión de la frontera bonaerense. Posesión y propiedad de la tierra en Chascomús, Ranchos y Monte. 1780-1880, Quilmes, 2005.

Revista de historiografía 26, 2017, pp. 67-83 
Como es de imaginar, este proceso apuntando a abrirse paso en otras zonas, solía ser iniciado por los hombres mientras las esposas permanecían al cuidado de la explotación familiar. Por otra parte, las guerras de independencia y guerras civiles que cubrieron la mayor parte del siglo representaron, como es previsible, una mortalidad más importante entre los hombres que entre las mujeres, con la obvia consecuencia de llevar más mujeres a encontrarse al frente de la unidad. Esta tendencia continúa aún en $1869^{19}$ donde, si bien no podemos identificar con certeza los jefes de familia, sí constatamos que la proporción de viudas nacidas en Argentina no solo aumenta con el paso del tiempo si no es superior a la de las viudas de origen europeo $^{20}$, lo que nos lleva a inferir que muchas de ellas se encontrarían igualmente con la familia bajo su responsabilidad.

Otro factor a tener en cuenta para explicar este importante aumento de unidades encabezadas por mujeres es el de cierta liberalidad en las relaciones sexuales que habría ido aumentando a lo largo del tiempo y que se habría traducido en una fracción de mujeres que de esa manera se encontraban asumiendo solas la crianza de sus hijos, como probablemente habrá sido la situación de la mencionada María Quiroga, madre soltera y jefa de familia en $1815^{21}$.

En el caso de las viudas contamos con algunos indicios a propósito de las soluciones que podían adoptar en caso de encontrarse al frente de la explotación, si bien estas soluciones no se prestan a conclusiones simplistas. Por una parte, porque se intuye claramente que las sucesiones registradas ante el notario solían esconder otros acuerdos entre los herederos que no podemos verificar, pero sobre todo, porque constatamos que una viuda con hijos menores podía optar por soluciones completamente opuestas. Como es bien sabido, el reparto sucesorio implicaba que el esposo supérstite conservara la mitad de los bienes gananciales -los acumulados durante el matrimonio-, mientras que los bienes propios del difunto, se dividían entre los descendientes. Constatamos que, mientras algunas de ellas declaraban que preferían

19. C. Contente y M. F. Barcos, "Un mundo rural en transición. La campaña bonaerense según el Primer Censo Nacional de Población de la República Argentina (1869)" Quinto Sol, Vol. 20, No 1, enero-abril 2016, 1-32. DOI: http://dx.doi.org/10.19137/qs0865 y C. Contente, “De los números a las personas. Los habitantes de San Vicente (Buenos Aires) según el Primer Censo Nacional Argentino (1869)”, Revista de Indias, (2017, núm. 269, 199-238. DOI: doi:10.3989/revindias.2017.007).

20. En San Vicente 18,3\% de las argentinas mayores de 14 años son viudas, mientras que 13\% de las extranjeras lo son. Esta tendencia se registra igualmente en las otras zonas estudiadas de la provincia de Buenos Aires, cf. C. Contente y M. F. Barcos “Un mundo rural...” loc.cit. Recordemos que los extranjeros estaban eximidos del servicio militar.

21. Cf. J. L. Moreno, "Sexo, matrimonio y familia: la ilegitimidad en la frontera pampeana del Río de la Plata, 1750-1850", en Boletín del Instituto de Historia Argentina y Americana N¹6-17, 1998; Historia de la familia en el Río de la Plata, Buenos Aires, 2004, 97-122; "El matrimonio y la vida familiar en el escenario de la Buenos Aires colonial”, en R. Fradkin, (dir.) Historia de la Provincia de Buenos Aires, T.II: Buenos Aires de la conquista hasta 1820; Buenos Aires, 2012, 210-237; M. C. Cacopardo y J. L. Moreno, "Cuando los hombres estaban ausentes : la familia del interior de la Argentina decimonónica” en H. Otero y G. Velázquez (comp.), Poblaciones Argentinas. Estudios de demografía diferencial, Tandil, 1997, 13-28; D. Santilli, Quilmes : Una historia social. I - Desde la reducción hasta la caída del rosismo. La historia vista desde los pobres, Buenos Aires, 2012, 100-106. 
conservar para sí mismas los bienes más «seguros» como las tierras a fin de poder obtener dinero en efectivo para los hijos más fácilmente vendiendo el ganado, otras optaban por hacer atribuir las parcelas a los menores y conservar ellas el ganado, carretas y demás recursos que les permitirían seguir desarrollando las actividades productivas para mantener la familia ${ }^{22}$. Sea cual fuera la solución adoptada ante el escribano, está claro que ellas continuaban con la explotación que sería repartida entre los herederos llegado el momento oportuno.

En fin, si más allá de la necesidad de ganarse el pan, desde un punto de vista jurídico, la capacidad de acción de las viudas era equivalente a la de un hombre a la hora de tomar decisiones o firmar un contrato -en particular tras haber recibido la tutela de los hijos que, en términos generales, le otorgaba el juez- éste no era el caso de las mujeres casadas.

Como decíamos más arriba, si el matrimonio representaba seguridad, estabilidad y protección para las mujeres, esta condición implicaba igualmente, -en teoría al menos- un status de completa sumisión y dependencia con respecto al marido: era una sociedad patriarcal y paternalista, según los cánones propios de la época. Sin embargo sabemos, como decíamos, que esto no era del todo así. Como ya señalara Silvia Mallo, las culturas e ideales familiares, conocían en la práctica diferentes matices según los diversos medios sociales ${ }^{23}$. Esto era válido para los sectores más altos de la sociedad, pero no lo era tanto entre los sectores medios y bajos. Probablemente existían igualmente matices entre el medio rural y urbano, e incluso tal como hemos podido constatar en otros ámbitos relacionados con el aspecto formal de la transmisión de bienes, entre zonas de la campaña de antigua colonización y de frontera ${ }^{24}$. Veamos otros aspectos de la cuestión.

\section{El marco jurídico}

En la campaña de Buenos Aires, al igual que en el conjunto de América hispana, regía la legislación castellana, heredera de la tradición jurídica ligada al Derecho Romano y al Código Justiniano ${ }^{25}$. Esta legislación estaba basada en un conjunto de compilaciones de normas y leyes, entre las que tenían particular vigencia las Siete Partidas de Alfonso X (1256-1265) y las Leyes de Toro $(1505)^{26}$. Mientras las Partidas establecían que las mujeres podían ser excusadas por

22. A modo de ejemplo, los menores heredan casi exclusivamente tierras en las sucesiones de Guillermo López (AGN, Suc. 6510, 1845), o José de la Rosa (AGN, Suc. 7785, 1823); mientras que reciben animales, alhajas y bienes «más fácilmente reducibles» en el caso de Sebastián Acosta (AGN, Suc. 3475, 1818), Antonio Salomón (AGN, Suc. 8414, 1786), o Tomás Transmontano (AGN, Suc. 8558, 1787).

23. S. Mallo, "La mujer rioplatense..." loc. cit

24. C. Contente, “Tradiciones hereditarias bajo influencias: tres pueblos de la campaña bonaerense entre los siglos XVIII y XIX”, en Revista de Demografía Histórica, XXVII, II, 2009, segunda época, 53-76.

25. V. Tau Anzoátegui, Esquema histórico del derecho sucesorio del medioevo castellano al siglo XIX, Buenos Aires, 1982.

26. Este cuerpo de leyes fue completado o modificado en algunos aspectos, todo lo cual se encuentra recogido en la Novísima Recopilación de las Leyes de España (1805). 
no conocer las leyes ${ }^{27}$, las Leyes de Toro fueron más lejos aún en su intención de proteger su presunta fragilidad, precisando que cuando estuvieran casadas se requeriría la autorización del marido para celebrar contratos, presentarse a juicio, aceptar o repudiar herencias, etc. ${ }^{28}$. En este sentido, el matrimonio representaba una sociedad entre dos socios que no gozaban de los mismos derechos ni tenían las mismas capacidades.

Estos aspectos se fueron manteniendo en los sucesivos cuerpos jurídicos y pervivieron hasta bien entrado el siglo XX en la actual Argentina, ya que Dalmacio Vélez Sarsfield los incluyó en el Código Civil que se sancionó en 1870.

Sin embargo, aunque la legislación basándose en su fragilidad, previera protegerlas, al menos durante el periodo colonial y las primeras décadas del periodo independiente, ellas tenían de hecho cierta capacidad para emprender actividades económicas y según podemos entrever en algunas fuentes, podían incluso disponer de sus bienes propios más o menos libremente. Ya hemos mencionado a María Quiroga y a Mercedes Ávila, criadoras establecidas por su cuenta que por cierto, al ser solteras, gozaban de ciertas prerrogativas que, como decíamos, al menos desde el punto de vista de la legislación vigente, no estaban permitidas a las mujeres casadas.

Beatriz Ferreira, en cambio, era casada, y su historia apunta igualmente a la capacidad que podían tener algunas mujeres en estas áreas rurales, incluso siendo casadas, para disponer de sus bienes. Tras la muerte de Beatriz (1776), estallan conflictos entre Jacinto Silva, el viudo, y sus hijos que le reclamaban que declarase e incluyera en la división de bienes la dote que ella había recibido cuando se casó ${ }^{29}$. Jacinto, explica en un alegato muy colorido las circunstancias de su boda. Cuenta que fue a buscar esposa a Córdoba, que un amigo ofició de casamentero para negociar la unión y sus condiciones y que se casó sin realizar amonestacio$n \mathrm{~s}^{30} \mathrm{ni}$ haber visto antes a la que sería su esposa. Cuenta igualmente que cuando se casó, su esposa era muy pobre y «estaba muy desnuda» y que en el camino al lugar donde residirían de casados, se dio cuenta de que los acompañaba una mulata que resultó ser un regalo de su suegra para la esposa con motivo de la boda y que Beatriz la vendió en su ausencia por $300 \$$ con lo que se compró otra esclava, agregando que la difunta gastó el dinero que sobró según su conveniencia sin que él tuviera conocimiento ni la menor participación en estas transacciones. Aunque Jacinto sin duda orienta su relato según su conveniencia, para evitar que se le obligue a devolver ese dinero a sus hijos, da por sobrentendido en su discurso que ella tenía la libertad de disponer de sus bienes sin pedir su autorización y ni siquiera rendirle cuenta alguna, lo que no parece sorprender a la asistencia ni suscitar comentario de ningún tipo.

27. «los nuestros caballeros más se deben trabajar en el uso de armas que en aprender leyes; o si fuese muger, o menor de veinticinco años o labrador simple bien se pueden excusar en tales razones como estas, diciendo que no sabían las leyes», Partida V, Tit.14, ley XXXI.

28. Leyes de Toro LIIII a LX, Nueva Recopilación de las Leyes de España, Libro 5, título 3.

29. AGN, Sucesión 5871, 1776.

30. A partir del Concilio de Trento (1545-1563) las proclamas solo podían ser suspendidas en casos graves que las justificaran, D. Rípodas Ardanaz, El matrimonio en Indias. Realidad social y regulación jurídica, Buenos Aires, 1977, 79-81. 
Otro ejemplo concreto es el de Narcisa Ortega que en la década de 1840 también llevaba adelante sola su explotación en San Vicente. En 1815, ella vivía con su familia, en ese entonces encabezada por su padre, Julián, su segunda esposa y hermanos de ambas uniones. Cuando Julián falleció, en 1835, tenía 17 hijos. Narcisa fue quien se dirigió a las autoridades correspondientes a fin de dar inicio a los trámites sucesorios. Tras el reparto de bienes, los seis hermanos mayores, descendientes de su primer matrimonio -y entre los que se contaba Narcisa-, conservaron su explotación mientras los de la segunda unión recibieron en herencia algunos muebles y ganado. En el expediente Narcisa y dos de sus hermanas mujeres constan como solteras. Las tres seguirán viviendo en la explotación que encabezará Narcisa, de 48 años: aunque tenía hermanos varones, fue ella quien tras la muerte de su padre, se hizo cargo de la explotación familiar en la que residieron sus hermanas al menos hasta casarse. Narcisa se encargó igualmente de comprar a los otros herederos las fracciones de la propiedad que les había correspondido por herencia. El censo de 1838 nos la presenta al frente de su unidad en la que vive con otras cinco personas. Cuando falleció, unos 20 años después que su padre, seguía sola al frente de su explotación, sin embargo en ese entonces consta que está separada de su marido. En el testamento ${ }^{31}$ declara el destino que quería dar a sus bienes, y enumera las deudas contraídas, quedando en claro que gestionaba sola la propiedad. No sabemos cuándo se casó ni qué la llevó a separarse del marido, que la sobrevive y se hace representar en los autos sucesorios, pero evidentemente, la existencia del marido o de hermanos varones no fue un impedimento para que ella administrara sus bienes y llevara adelante su explotación de manera autónoma.

Otra de estas mujeres que figuraba casada en 1815 y como responsable de su explotación en 1836 es doña Josefa Pavón, esposa de José de la Rosa. Es un caso un tanto especial, porque aunque el censo de 1836 la presente sola y como jefe de familia de la unidad, sabemos que Josefa se había casado en 1832 con un jornalero mucho más joven que ella, que tenía la edad de sus hijos y que no aportó prácticamente bienes al matrimonio... Quizás porque es ella quien impone respeto en la familia o porque los suyos son bienes que han sido adquiridos durante el matrimonio anterior, el censista obvia la existencia del marido tanto en 1836 como en el censo ganadero de $1839^{32}$. Evidentemente es Josefa quien lleva adelante la explotación y el censista no hace más que poner por escrito lo que la comunidad percibe: ella es el jefe del hogar. Cuando Josefa falleció, en 1843 dejó estipulado en su testamento como quería que se repartieran sus bienes entre sus hijos y mejoró a sus dos hijas menores y solteras con el quinto de sus bienes. Una de ellas, Encarnación, se casó con su viudo, lo que permitió evitar la división de la propiedad principal. Lo notable es que con el correr de los años, veremos a Encarnación emprender por su cuenta arduas acciones legales para recuperar la fracción de tierras que tenía en propiedad su hermano fallecido, heredadas de sus padres sin que conste necesariamente que contaba con «la venia» de su marido para realizar tales operaciones. La veremos igualmente, ya mayor, dirigirse en varias oportunidades al escribano para dejar constancia de sus bienes y de diversas cuestiones relacionadas con estos. Claramente,

31. AGN, Sucesión 7284, 1856.

32. AGN Sala III - 33-4.7 
el hecho de estar casada, tampoco impidió a Encarnación promover las acciones en justicia convenientes para consolidar el patrimonio familiar.

Por otra parte, el derecho escrito autorizaba a las mujeres a acudir a los tribunales a denunciar a sus maridos en ciertos casos como malos tratos o falta de alimentos, derecho que no se privaron de ejercer ${ }^{33}$. Podían igualmente testar sin la intervención del marido. En ese sentido, hemos podido verificar en testamentos redactados por matrimonios en un corto intervalo de tiempo, como mientras uno de los esposos favorece a uno o varios de los hijos, el cónyuge toma otras disposiciones o agrega elementos a la declaración (como bienes aportados al matrimonio) que a la hora del reparto podían modificar seriamente el resultado de la operación prevista por quien había testado primero ${ }^{34}$.

Resumiendo, en lo que respecta a la gestión de sus bienes, las mujeres casadas disponían de un mayor margen de acción que el previsto por la ley y lo utilizaban: si, a diferencia de las solteras, en teoría gozaban de derechos equivalentes a los un menor de edad y necesitaban la autorización del marido para tomar decisiones o emprender cualquier acción, (incluso si esa decisión involucraba bienes de su exclusiva propiedad, como los heredados de sus padres), en la práctica esto no era tan estricto como se podría presumir partiendo exclusivamente de la legislación escrita: como vimos a través de los casos mencionados, al menos hasta mediados del siglo XIX, la documentación podía eventualmente omitir la «venia»o autorización expresa del marido ${ }^{35}$, el marido mismo puede mencionar que ella dispuso de sus bienes sin consultarle o ella podía, haciendo uso de sus derechos, redactar un testamento que no seguiría necesariamente las líneas del que haría el propio marido.

En conclusión, hoy en día tendemos a menudo a considerar exclusivamente los derechos y obligaciones previstos por el derecho escrito, cuando en la práctica, éste podía no ir en el mismo sentido que la costumbre con el cual, según expresan las Partidas, se conjugaba: «Dos raíces son aquellas de que nace el derecho comunal, porque se guían y se mantienen las gentes en justicia y en concordia y en paz, la primera es la ley escrita, la segunda es costumbre antigua que vale tanto como ley, a la que dicen en latín consuetudo» ${ }^{36}$. En ese sentido, tal como indica un manual de derecho de la época: «Costumbre es: ley no escrita introducida por el uso. Para que sea tal, y no corruptela se requiere que el uso sea del pueblo o de su mayor

33. V. Kluger, Escenas de la vida conyugal. Los conflictos matrimoniales en la sociedad virreinal rioplatense, Buenos Aires, 2003; R. Cicerchia, Historia de la vida privada en la Argentina, Buenos Aires, 1998, 67-75; S. Mallo, "Conflictos y armonías: las fuentes judiciales en el estudio de los comportamientos y valores familiares" en D. Celton; M. Ghirardi, A. Carbonetti (coord.), Poblaciones Históricas. Fuentes, métodos y líneas de investigación, Serie de Investigación no 9, Río de Janeiro, 2009, 387-401.

34. Ver por ejemplo los testamentos del matrimonio formado por Agustín Reyes y Luisa Ortega (AGN, Suc. 7828, 1859) o los de Matías y Josefa Rodríguez, AGN, Suc. 7806, 1850).

35. Al caso que mencionamos de Encarnación de la Rosa, podríamos agregar otros como por ejemplo el de Luisa Ortega de Reyes que participa en los autos sucesorios de su padre sin que conste la autorización de su marido.

36. Partidas, Título II.

Sobre Usos y Costumbres ver V. Tau Anzoátegui, El poder de la costumbre. Estudios sobre el Derecho Consuetudinario en América hispana hasta la Emancipación, Buenos Aires, 2001. 
parte, por el espacio de diez años, sabiéndola el jefe de la tierra y no contradiciéndola (P1., Tit.2.1.5) y que esté en armonía con la utilidad general. Dos juicios ó sentencias uniformes, son una de las pruebas de la costumbre. La costumbre deroga la ley anterior que le sea contraria, e interpreta la dudosa. (P.1 tit. 2.1.6)

La redacción del Código tuvo la clara y decidida intención de acabar con usos y costumbres, de racionalizar, sistematizar y fijar formalmente el conjunto de leyes, con el que se procuraba dar garantías jurídicas que ofrecieran certeza y seguridad al conjunto de la población ${ }^{38}$, suprimiendo las «desviaciones» y libres interpretaciones que habilitaba la costumbre. Probablemente en la práctica y en la medida en que su aplicación fuera efectiva, el Código habrá representado una limitación concreta para el margen de movimiento del que de hecho disponían las mujeres.

\section{Algunas conclusiones}

No hay duda alguna de que las mujeres formaban parte del mundo del trabajo rural y que frecuentemente debían trabajar para participar en el sustento familiar -o asumirlo solas-. El estado civil en que se encontraran, la cargas familiares que llevaran, la sociedad e incluso las condiciones que ofrecieran el lugar donde se encontraban, marcarían las pautas de su margen de acción. En todo caso, está claro que las solteras tenían desde el punto de vista jurídico una amplia capacidad para actuar, que de hecho algunas ejercían en esta zona rural cuya sociedad sin duda era, tal como hemos visto, menos estricta que otras regiones del antiguo virreinato. Sin embargo, difícilmente habrán sido capaces de reunir solas y por sus propios medios bienes materiales que justificaran la redacción de un testamento ante notario o un procedimiento sucesorio.

En cuanto a las mujeres casadas, estaban menos expuestas que solteras y viudas a la necesidad del trabajo remunerado fuera del hogar, aunque unas vez viudas debían asumir formalmente la responsabilidad de la unidad familiar y de su sustento.

La estrategia a adoptar en ese caso estaría directamente relacionada con las circunstancias en que se encontrara cada una, -la composición de la familia (la edad y capacidad de los hijos, la presencia en la unidad de otros miembros fueran o no de la familia, de esclavos, de jornaleros...), la red de familiares y amigos en la que se pudiera apoyar, el acceso a la tierra, etc.,- Distintos factores determinarían la estrategia a seguir: ¿integrarse en la unidad de algún pariente o amigo? ¿continuar con la explotación para poder luego transmitirla a los hijos? Si las circunstancias eran favorables, sería el ideal, y tenían la capacidad para hacerlo: sin duda participaban ya junto a sus maridos en las tareas y gestión de la explotación, participación que a menudo tendría lugar desde la sombra o, como hemos visto, tomarían sus propias iniciativas

37. Cf. J. E. de Ochoa, Manual del abogado americano, Lima, 1834, 2-3

38. A. Guzman, Historia de la Codificación civil en Iberoamérica, Fundación Histórica Tavera, 2000, p.164; V. Kluger, "Recopilación, codificación, descodificación y recodificación. Permanencias y cambios en el derecho de familia argentino”, en O. Cruz Barney, (coord.) La codificación, México, 2006, 77-102. 
sin que el marido otorgara formalmente el consentimiento escrito que correspondía ante el escribano y que muy probablemente nadie les reclamaría como condición para tratar con ellas.

Si bien en algunos aspectos como los relacionados con la propiedad y su uso, la pervivencia de la costumbre y las tensiones que generaban las prácticas agrarias locales y los intereses privados son palpables ya desde las décadas que precedieron la promulgación del código $^{39}$, en otros aspectos como los analizados aquí, la práctica se vuelve más escurridiza, estamos ante comportamientos espontáneos que solo algunos indicios nos permiten detectar. Es así como cuando analizamos cuidadosamente algunos documentos, solemos encontrar actos o gestos que no se corresponden con lo que preveía la ley escrita: hasta mediados del siglo XIX algunas mujeres por ejemplo, recibían una herencia o realizaban algún acto ante escribano, sin que constara necesariamente la «venia» y firma del marido autorizándola... ¿se trata de negligencia del escribano? Intuimos que más probablemente refleja el indicio de una realidad concreta que existía de hecho, si bien no de derecho, que las mujeres podían incursionar en ese terreno que se suponía masculino realizando contratos, participando en la administración de bienes, vendiendo o comprando dentro del marco de un consenso social y sin que fuera siempre indispensable la intervención o intermediación del marido, sin que la oportuna «venia» se incluyera necesariamente en las actas.

En fin, en una sociedad básicamente oral estos pequeños episodios o detalles mencionados al descuido nos dejan entrever algunas prácticas que eran tan corrientes que ni merecía la pena mencionar, así entendemos que aunque la ley escrita previera que las mujeres casadas se desentendieran de los aspectos relacionados con la gestión del dinero, en la práctica, este ideal tradicional de la mujer sumisa cuyo marido administraba los bienes comunes y propios según su voluntad, presenta aquí, en el mundo rural, algunos matices: según Jacinto Silva, su mujer había vendido su esclava y comprado otra sin que él se enterase, Josefa Pavón o Encarnación de la Rosa emprendieron acciones judiciales por su cuenta y Mercedes Ávila, siendo soltera -lo que no implicaba limitaciones jurídicas pero sí alguna «incomodidad» social-, estaba al frente de una explotación que incluía peones, igual que luego su sobrina Narcisa, separada, llevaba adelante su explotación y se ocupó de recuperar entre sus hermanos fracciones de la propiedad heredada de su padre sin la intervención del marido, en un lugar que, según hemos visto no siempre resultó tan subordinado como se presumía. Cabe destacar igualmente la total falta de protección en la que se encontrarían las mujeres ilegítimas y madres solteras, un sector que alcanzó proporciones considerables en la campaña bonaerense, y que han dejado muy pocas huellas en los documentos ${ }^{40}$. No ponemos en duda que se tratara de una sociedad paternalista, pero en la práctica y en estos ámbitos concretos, los roles y esferas de poder entre hombres y

39. B. Zeberio, "Derechos de propiedad y sistema normativo en la Argentina del siglo XIX”, en Economía, vol. XXIX, n 57-58, junio-diciembre de 2006, 233-270 y "Los hombres y las cosas. Cambios y continuidades en los Derechos de propiedad (Argentina, siglo XIX)” en Quinto Sol no 9-10, 2005-2006, 151-183 y V. Tau Anzoátegui, El poder... op.cit., 54-61.

40. H. Otero estima que para principios del siglo XX un nacimiento cada cinco era ilegítimo. H. Otero, Estadística y... op. cit. 2da parte. Siendo el sector más frágil y marginal de la sociedad no solían tener bienes ni pasar ante el escribano para dejar constancia de sus voluntades. 
mujeres comportaban matices y no estaban tan claramente delimitados como lo pretendía el ideal al que se aspiraba. En fin, la realidad hacía que el sistema no fuera tan rígido y que las mujeres, estuvieran o no casadas, incursionaran en territorios supuestamente reservados a los hombres y que de hecho tuvieran capacidad para decidir sobre sus bienes o tomar decisiones en terrenos que les estaban supuestamente vedados.

Cuando entró en vigencia el Código Civil (1870), algunas cuestiones como la ilegitimidad y el destino de hijos naturales e ilegítimos o su capacidad para heredar fueron objeto de largas discusiones y acalorados debates entre legisladores y juristas, mientras que la cuestión de las capacidades y derechos de las mujeres casadas como aquellos relacionados con la administración de sus bienes, permanecieron sin cambios, sin que se suscitaran oposiciones o debates ${ }^{41}$. Evidentemente, no era éste un tema de actualidad en aquel entonces.

La promulgación del Código Civil abre algunos interrogantes, ¿El Código conoció una aplicación efectiva en este ámbito? ¿Representó una limitación concreta para las mujeres de los sectores medios o subalternos? ¿Indujo a alguna adaptación en los comportamientos? Son algunas de las pistas que nos proponemos investigar en un futuro próximo.

41. B. Paz, Discusión de la fe de erratas y correcciones al Código Civil propuestas por el Senador de Tucumán, Benjamín Paz y la Comisión de Lejislación, Cámara de Senadores de la Nación Argentina, Buenos Aires, 1879 . 